\title{
An examination of direct selection typing rate and accuracy for persons with high-level spinal cord injury using QWERTY and default on-screen keyboards
}

Michele Hurlburt, MS, OT(C) and Kenneth J. Ottenbacher, PhD, OTR

School of Occupational Therapy, Dalhousie University, Halifax, Nova Scotia B3H $3 J 5$ Canada;

School of Health Related Professions, State University of New York at Buffalo, Buffalo, NY 14214

\begin{abstract}
A single-subject rapid alternating treatment design with replication was used to compare the efficiency of two keyboard layouts, QWERTY and default, for persons with high-level spinal cord injury. The LIAISON system and proportional-drive chin controller provided computer access/writing to four subjects. Three efficiency characteristics were examined: keystrokes per minute, keystroke accuracy, and keystroke corrections. Baseline sessions were followed by 10 to 12 alternating treatment sessions for all subjects. Each alternating treatment session involved six text entry trials-three text entry trials using the QWERTY keyboard layout and three using the default keyboard layout. Four follow-up sessions were completed for each subject using the default keyboard configuration. Data analysis revealed that the default keyboard layout provided greater keystrokes per minute for two of the subjects. The keystroke accuracy was between $98 \%$ and $100 \%$ for all subjects on both keyboard layouts. The findings of this investigation revealed no clinically significant difference in typing performance across the two keyboard layouts for the participating subjects. The results also indicate that previous experience with a keyboard configuration is an important factor in determining performance. A possible treatment interaction or carryover effect between the two keyboard configurations was noted and has implications for the type of single-subject design used in future investigations.
\end{abstract}

Address all correspondence and reprint requests to: Kenneth Ottenbacher, PhD, SUNY at Buffalo, 435 Kimball Tower, Buffalo, NY 14214.
Key Words: assistive technology, computers, singlesubject research, spinal cord injury.

\section{INTRODUCTION}

Computer-related technologies hold great potential for influencing the independence and lifestyles of people with disabilities (1). Computerbased, alternative-access key entry and writing systems can reduce the number of barriers in the home, community, and workplace that limit independent function and productive employment. The efficiency of computer-assisted communication systems is partially determined by how quickly text can be entered (2). Text entry efficiency is dependent on the alternative access interface, and the quality of interaction between the user, the interface, and the selection method $(3,4)$. The computer keyboard layout is also an important variable in this process, affecting speed, accuracy, and ease of learning (5). The argument has been made that the standard QWERTY computer keyboard may be difficult for individuals with sensory and motor disabilities to operate (6). Alternative keyboards may prove to be useful for improving and/or maximizing the writing performance of an individual. For example, the Dvorak keyboard arrangement is one popular alternative to the standard QWERTY keyboard (7). 
Another keyboard layout referred to as the default has been developed by Bayer and associates as part of a comprehensive, computer-based, alternativeaccess communication system called the LIAISON (8).

The LIAISON is a keyboard emulator providing individuals with high-level spinal cord injury (SCI) with alternative keyboard layouts and a control interface that is adaptable to their motor limitations. The LIAISON with the proportionaldrive chin controller and default keyboard is reported to provide users with a convenient method to enhance typing speed and accuracy. According to Bayer, et al. (8), the default keyboard increases typing speed, compared with the QWERTY layout, by minimizing the distance between the cursor movements when typing typical text. This is a logical assumption in view of the fact that the standard QWERTY keyboard layout has been frequently criticized for being inefficient $(9,10)$ and reportedly designed to slow down the typist $(7,10)$.

The purpose of this study was to assess keystroke selection efficiency of the default and QWERTY keyboard layouts using the proportional chin controller as an input device with the LIAISON system. The study was designed to test the assertion of Bayer, et al. that the default keyboard used in conjunction with the LIAISON system would provide superior keystroke performance when compared with the standard QWERTY keyboard. Keystroke selection performance characteristics included keystroke rate, accuracy, and number of corrections.

\section{METHODS}

\section{Subjects}

Four individuals with a wide variety of functional ability and keyboard experience were invited to participate in this study. Three of the individuals had quadriplegia due to a high-level SCI with lesions at $\mathrm{C} 4$ or higher and required a writing system. The fourth individual was nondisabled.

Subject 1, a 29-year-old man with a C4 SCI, was 9 years 10 months postinjury at the time of the study. He had used the LIAISON with the default keyboard for 8 years. He was employed through a local bank as a computer programmer/analyst and worked from his home via modem. He used his
LIAISON as a writing system for programming approximately 8 hours a day.

Subject 2, a 33-year-old man with a C4 SCI, was 7 years 9 months postinjury at the time of the study. He had used the LIAISON with the default keyboard for 4 months, 2 to 3 hours a day. He was a college student pursuing an associate's degree in data processing and used his computer for programming and completing written assignments.

Subject 3, a 38-year-old man with a C2-4 incomplete $\mathrm{SCI}$, was 2 years 7 months postinjury at the time of the study. He used Free Board with the QWERTY layout in combination with Vocal Link (voice input) as a writing system. He had no experience with the LIAISON prior to this study. He was employed 10 hours a week at a local community living center.

Subject 4, a 27-year-old nondisabled man, was a self-taught QWERTY keyboard typist. He had used a QWERTY computer keyboard for 5 and one-half years. He was a full-time graduate student working on a Ph.D. in chemical engineering.

These four individuals represented a wide range of physical ability and keyboard performance. This variability in functional capacity and experience was necessary to enhance the sensitivity of the investigation to determine initial differences in performance between the two keyboard configurations. Any initial differences could then be examined using a larger sample of homogeneous subjects.

\section{Independent variables}

The default keyboard layout used in this study is based on the frequency of letter use and the frequency of letter patterns and sequences in the English language. As a result, the vowels A-E-I-OU-Y are found in the central columns. Most of the consonants are alphabetically ordered in a manner that supports commonly typed letter combinations $(10,11)$. The QWERTY layout used in this study is the traditional QWERTY keyboard in terms of the alphabetic arrangement of letters. The layouts for the QWERTY and default keyboards are presented in Figure 1 and Figure 2, respectively.

The LIAISON access system (see Figure 3) used in this study consists of the LIAISON electronic box and composite color monitor that are positioned alongside a computer and its original monitor. A full-color keyboard screen is displayed on the LIAISON monitor. 
The LIAISON is the only microcomputer-based system that provides direct selection input through a proportional-drive chin controller (see Figure 4). The chin controller is operated through small, precise neck and chin movements. The subject points to the desired character by positioning the cursor over it for a brief, user-defined acceptance time, thereby entering that character into the computer. This procedure is believed to be faster and less physically demanding than other methods (12). Another advantage of the chin controller is that it allows for greater independence in computer startup/operation (8).

The cursor movement sensitivity can be altered to suit the physical needs of the user by adjusting the chin controller sensitivity (left, right, up, down). For example, individuals with restricted movement or asymmetrical range of motion can adjust the sensitivity to make all areas of the keyboard accessible with minimum effort.

\section{Dependent measures}

An executable computer software program written in Pascal was used to collect the data on keystroke input. This computer program recorded time to the nearest tenth of a second; the number of keystrokes including all punctuation, spaces, shifts, and letters; and the number of backspaces used for each trial. The information from each trial was printed to the screen and saved to disk as the subjects ended the trial. The data were integrated into three dependent measures: 1) keystrokes per minute (KSPM); 2) keystroke accuracy (KA); and, 3) keystroke corrections per minute (KC).

KSPM was calculated for each trial by dividing the number of keystrokes by the duration of the trial. KSPM was converted to words per minute (WPM) in order to provide a functional measure of the performance of the individual. WPM was computed by dividing the number of KSPM by five, the standard letter-to-word conversion factor (13). This measure of WPM is comparable to WPM conversions in other studies reported in the literature $(14,15)$.

KA, defined as the percentage of correct characters, was calculated by dividing the number of correct characters by the total number of characters printed. KA data for each trial were obtained and analyzed from the hard copy printed on completion of each trial.

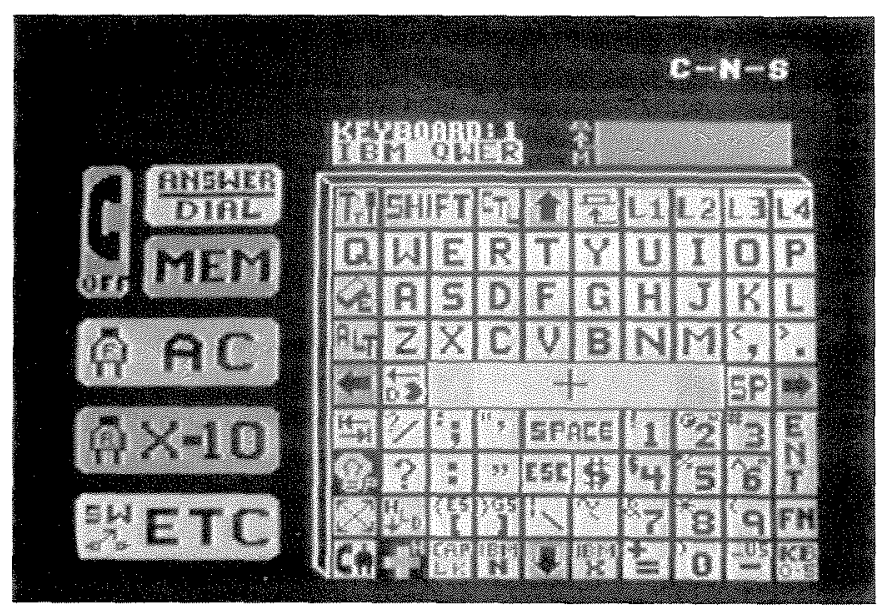

Figure 1.

The LIAISON QWERTY keyboard layout.

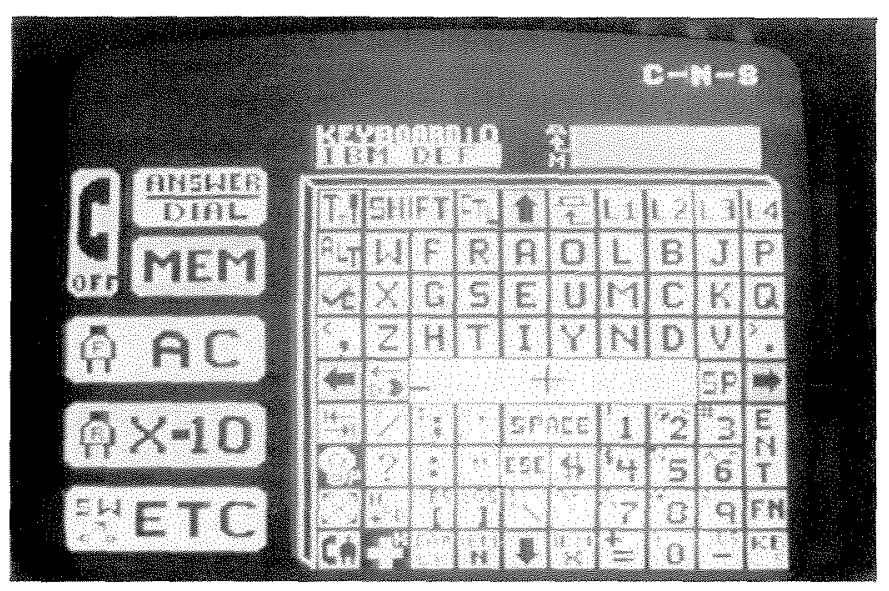

Figure 2.

The LIAISON default keyboard layout.

$\mathrm{KC}$ per minute was computed for each trial by dividing the number of backspaces by the duration of the trial, and reported in terms of backspaces per minute.

\section{Research design}

A single-subject, rapid alternating treatment design with replication was used to compare the keystroke performance scores between the two keyboard layouts for four subjects. The alternating treatment design allows for evaluation of two or more treatments administered in the same phase, with the purpose of determining which treatment is most effective. Unlike other designs, the interven- 


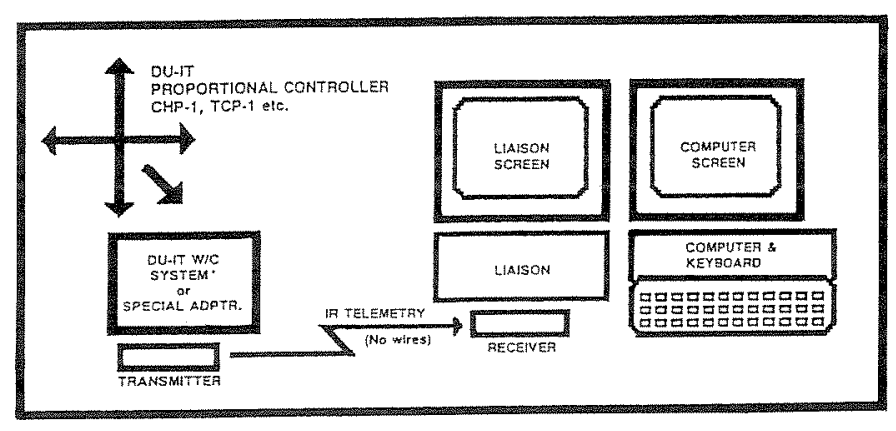

Figure 3.

The components of the LIAISON system.

tion can be implemented and evaluated even when baseline data show initial trends (16).

\section{Procedure}

This study was conducted with each subject in his "natural typing environment." For example, Subject 1 was seated in his powered wheelchair in front of his LIAISON system with the text placed in a comfortable viewing position. The environment and set-up remained consistent for each subject across all sessions.

An initial baseline phase consisting of four sessions with three trials per session was conducted over 14 days. A 15-minute keyboard warmup was followed by three 35 -word text trials. Keystroke performance scores were measured using the current keyboard layout (default or QWERTY) of the individual in order to establish a performance level for the experienced LIAISON users and a baseline skill level for the novice LIAISON users. This was followed by 10 alternating treatment sessions for Subjects 1 and 2 and 12 alternating treatment sessions for Subjects 3 and 4 .

Alternating treatment sessions. Each treatment session involved six 35 -word text entry trials, three using the default keyboard and three using the QWERTY. A 15-minute keyboard warmup was given prior to the introduction of each keyboard condition in each session. The keyboard conditions were systematically alternated so that each keyboard layout was presented to each subject in first and second order. The sessions were scheduled over a 6-week period with two sessions per week. Finally, a follow-up phase consisting of four sessions with three trials per session was conducted in order to separate out order and/or carry-over effects from the previous alternating treatment phase $(16,17)$. The default keyboard layout was used in the follow-up phase for all four subjects.

The subjects were instructed to type the 35word text trials as accurately and quickly as possible, using the backspace key to correct mistakes in the working word. However, they were not allowed to go back to correct previously unrecognized mistakes. The text for this study was chosen from a popular magazine article that was greater than 2,500 words. The text was divided into 96 segments of 35 words. The segments were randomly assigned across sessions to ensure that the text was consistent and of equal difficulty across the sessions/trials.

Each subject used a proportional chin controller as an interface. The LIAISON system and proportional chin controller were adjusted to allow each individual maximum control over the cursor. Each subject entered text via a word processor during the warmup and via the Pascal-based computer program for each text trial. The LIAISON settings were adjusted to suit Subjects 3 and 4, new users of the LIAISON system, prior to the baseline phase. The adjustment procedures outlined by the DU-IT Control Systems Group (11) in the LIAISON instruction manual were followed.

The LIAISON system allows the user to alter the acceptance time (i.e., the length of time the cursor must stay over a letter before sending the

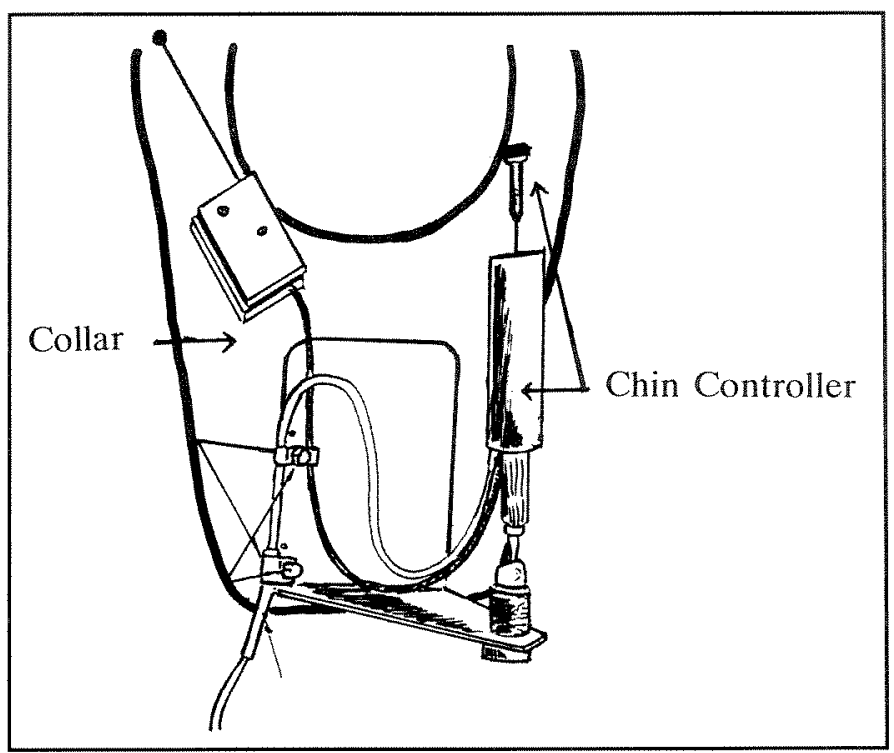

Figure 4.

The proportional-drive chin controller and collar. 
selected letter to the computer). A beep is incorporated into the acceptance time and provides the user with auditory feedback when a letter is sent to the computer. The acceptance time settings range from 1 to 16 , with 16 being the fastest. The calculated real-time value for setting 16 is 0.268 seconds. The acceptance time setting was increased systematically for each subject until he reached the maximum value of 16. Subject 1, an expert user, started at 16, his present acceptance value. Subject 2, a new user, started at an acceptance value of 12 . Subjects 3 and 4 , novice users, started at 8 . This was the acceptance setting determined as comfortable for them during the first baseline session warmup. For Subjects 2, 3, and 4 , the acceptance setting was increased by two levels prior to session three of the baseline phase and sessions three and six of the treatment phase, and again prior to treatment session ten for Subjects 3 and 4 . The criteria for increasing the acceptance time setting involved the comfort and skill of the subjects. For example, if the subject felt his performance was not being adversely affected (i.e., an increase in number of errors or a need to rest between keystrokes to avoid errors), the acceptance time setting was increased as scheduled.

\section{Data analysis}

The data from the three related text entry trials of each subject were averaged and graphed for visual analysis. The keystroke performance of each subject was compared between the keyboards.

\section{RESULTS}

Data from Subject 1 are presented in Figures 5a, b, and c. As an experienced LIAISON and default keyboard user, he demonstrated greater KSPM using the default (Figure 5a). His KSPM for the final treatment session converted to $13.61 \mathrm{WPM}$ on the default and 10.41 WPM on the QWERTY. His KA remained above 99 percent (Figure 5b) for both keyboards. Finally, he made few errors on either keyboard, indicated by his minimal number of backspaces and high accuracy rate.

Data from Subject 2 are presented in Figures $\mathbf{6 a}, \mathbf{b}$, and c. KSPM for this new LIAISON and default keyboard user was greater using the default keyboard throughout the study. He demonstrated an increase of 12 KSPM from the first baseline session
SUBJECT 1

KEYSTROKES PER MINUTE

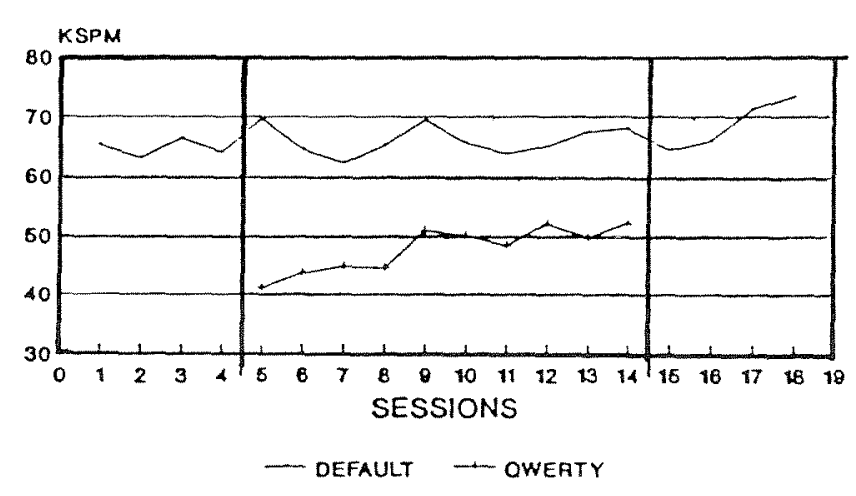

A

\section{SUBJECT 1} KEYSTROKE ACCURACY

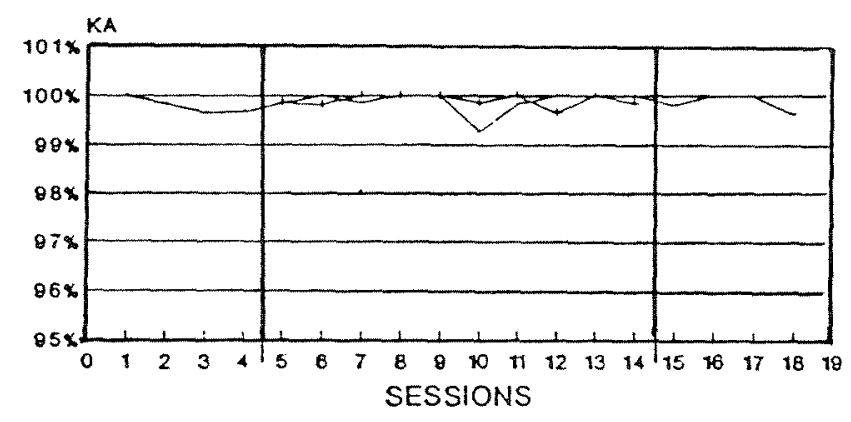

- DEFAULT - OWERTY

B
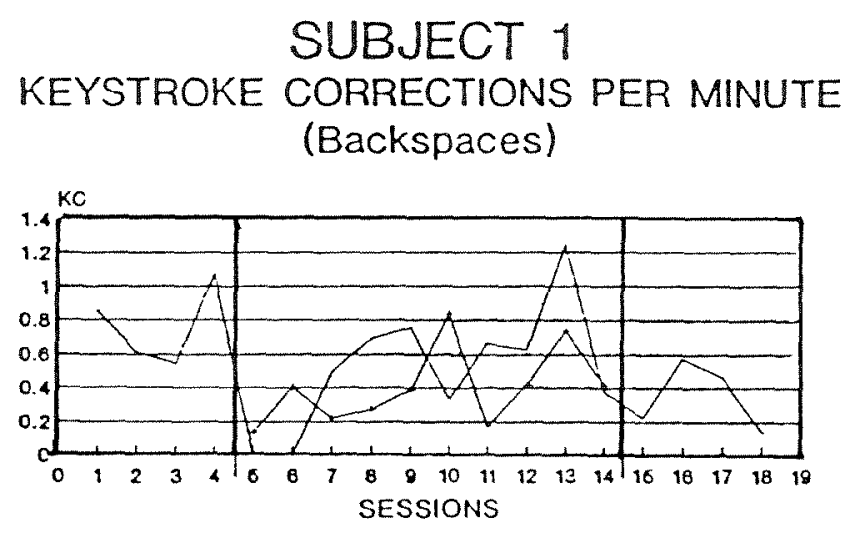

- DEFAult OWERTY

C

Figure 5 a, b, c.

Subject 1: Keystroke selection performance. 
SUBJECT 2 KEYSTROKES PER MINUTE

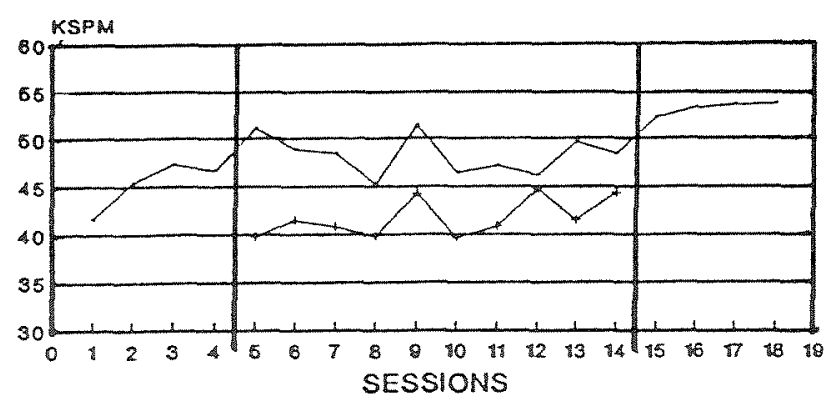

- oefault owertr

A

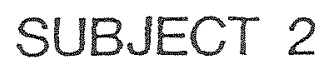
KEYSTROKE ACCURACY

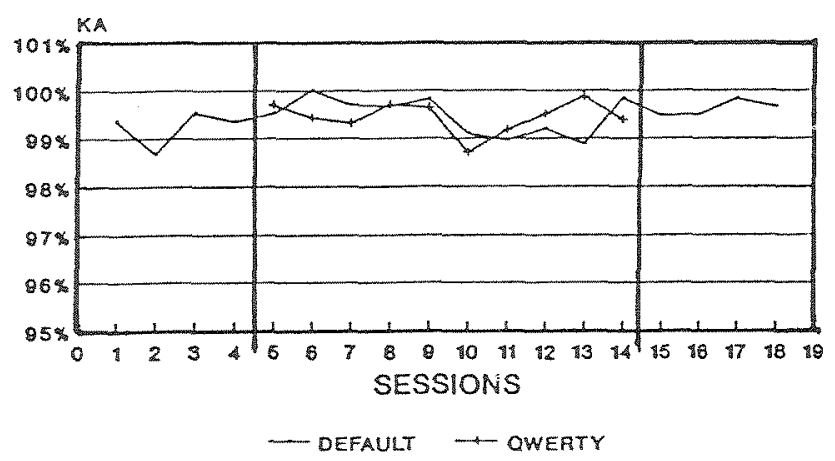

$B$

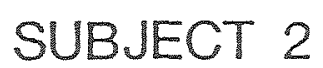

KEYSTROKE CORRECTIONS PER MINUTE (Backspaces)

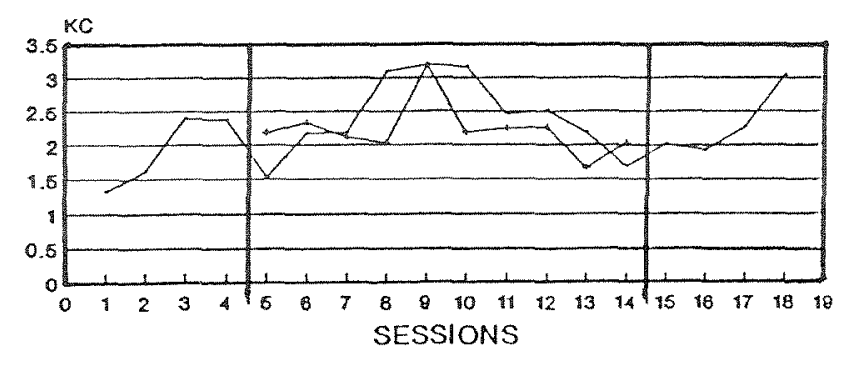

- defautr - awerty

C

Figure 6a, b, c.

Subject 2: Keystroke selection performance. to the last follow-up session. He reached 9.67 WPM by the end of the treatment on the default and 8.85 WPM on the QWERTY, a difference of 4.1 KSPM. His WPM on the default rose during the follow-up phase to 10.72 , an increase of 1.05 words or 5.25 KSPM (Figure 6a). His accuracy was similar on both keyboards, ranging from 98.88 percent to 100 percent on the default and from 98.71 percent to 99.87 percent on the QWERTY during the treatment sessions (Figure 6b). His $\mathrm{KC}$ varied across both keyboards, ranging from 1.53 to 3.18 for the default and 1.66 to 3.17 for the QWERTY during the treatment sessions (Figure 6c).

Data from Subject 3 are presented in Figures $\mathbf{7} \mathbf{a}, \mathbf{b}$, and $\mathbf{c}$. KSPM for this novice LIAISON user but experienced QWERTY keyboard user was greater using the QWERTY keyboard for all but two sessions in the treatment phase and two sessions in the follow-up phase. Generally, however, there was only a marginal visual separation between the KSPM for the keyboards. He achieved a maximum of 16.39 WPM on the QWERTY and a maximum of 16.02 on the default during the final treatment session. He demonstrated an increasing trend in KSPM for both keyboards, with a range of 35.38 to 81.93 on the QWERTY and a range of 49.68 to 83.79 on the default (Figure 7a). His accuracy was similar with both keyboards, ranging between 98.14 percent to 100 percent on the default and between 98.15 percent to 99.66 percent on the QWERTY (Figure 7b). His backspaces (KC) increased gradually, but in a similar fashion across both keyboards, with a maximum of 8.07 on the QWERTY and 6.13 on the default during the treatment phase (Figure 7 c).

Data from Subject 4 are presented in Figures $\mathbf{8 a}, \mathbf{b}$, and $\mathbf{c}$. KSPM for this novice LIAISON user and self-taught QWERTY user was marginally greater for the QWERTY keyboard until the eleventh treatment session, then marginally greater for the default until the completion of the study. There was not a definite separation in the KSPM across the two keyboards for Subject 4. He achieved 10.76 WPM on the QWERTY and 10.92 WPM on the default during the final treatment session. He demonstrated an increasing trend in KSPM for both keyboards, with a range of 42 to $55.03 \mathrm{KSPM}$ on the QWERTY and 41.88 to $57.81 \mathrm{KSPM}$ on the default (Figure 8a). He consistently obtained 100 percent or near 100 percent accuracy for both keyboards, 
SUBJECT 3 KEYSTROKES PER MINUTE

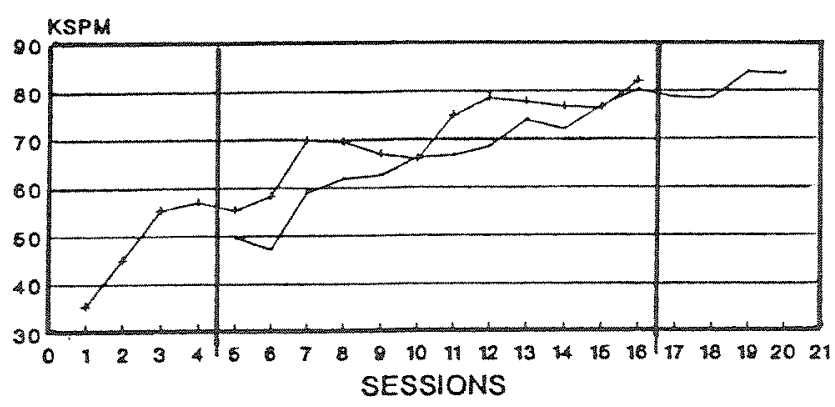

- DEFAULT + OWEATY

\section{A}

SUBJECT 3 KEYSTROKE ACCURACY

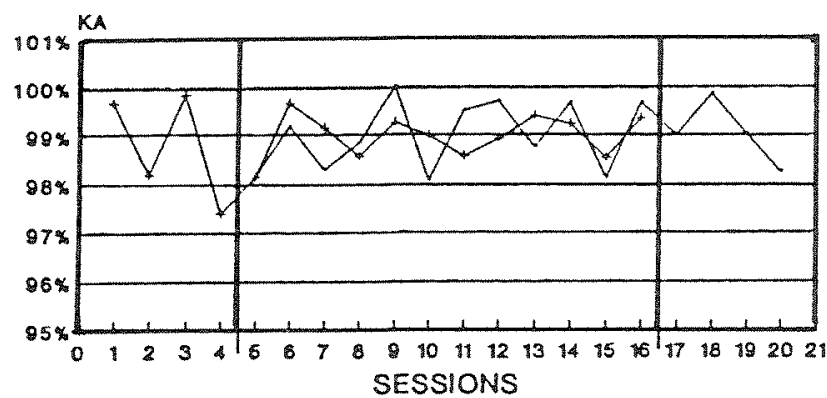

- DEFAULT $\rightarrow$ - OWERTY

$B$

SUBJECT 3

KEYSTROKE CORRECTIONS PER MINUTE (Backspaces)

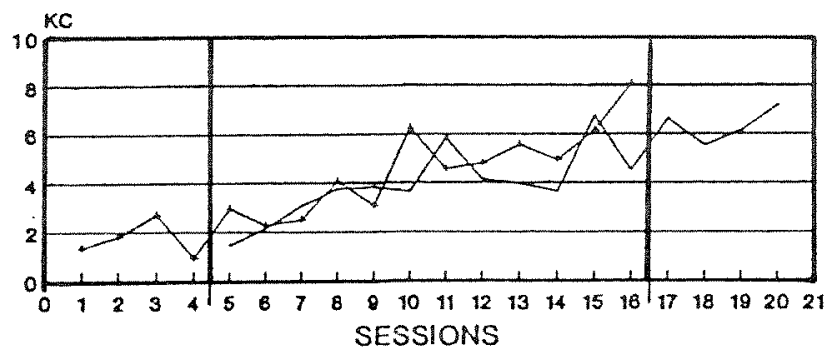

- DEFAulr $\rightarrow$ OWERTY

C

Figure 7 a, b, c. Subject 3: Keystroke selection performance.
SUBJECT 4 KEYSTROKES PER MINUTE

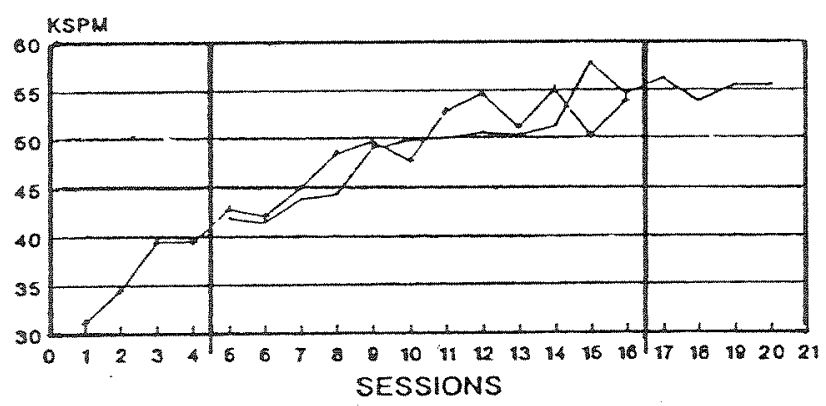

- default $\rightarrow$ oweatr

A

\section{SUBJECT 4} KEYSTROKE ACCURACY

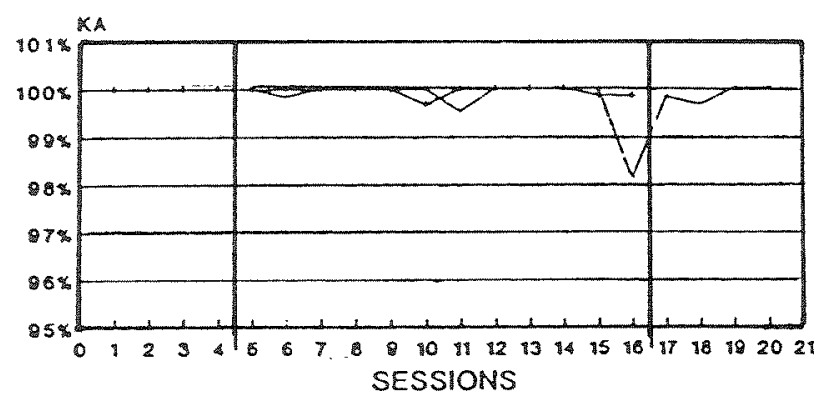

- Default $\rightarrow$ OWERT

B

\section{SUBJECT 4}

KEYSTROKE CORRECTIONS PER MINUTE (Backspaces)

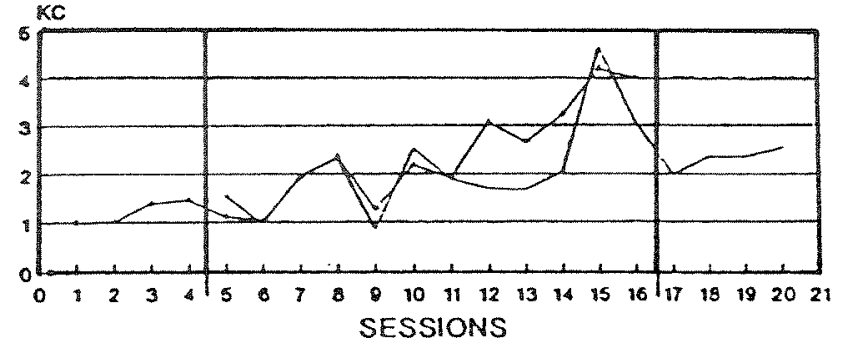

- oefaul - owertr

C

Figure $8 \mathrm{a}, \mathrm{b}$, c.

Subject 4: Keystroke selection performance. 
except in treatment session 12 when he omitted a word in a text trial with the default keyboard (Figure $\mathbf{8 b}$ ). His $\mathrm{KC}$ overlapped and then varied together throughout the treatment phase (Figure 8c).

Another factor affecting performance between the two keyboards which was not reflected in the graphed data is the physical distance traveled on each keyboard when entering text. Computations were performed to determine the letter transition distance for a set of 50 most frequently used words, based on length multiplied by frequency of use. According to Vanderheiden and Kelso (18) these 50 words account for 40-50 percent of the total words used in communication. The linear distance traveled between keys on each keyboard was measured for the word set. An analysis of variance indicated a statistically significant difference in the distance traveled when using the default keyboard layout compared with using QWERTY keyboard layout (see results in Table 1). Data in Table 1 demonstrate that the transition distance between letters was greater with the QWERTY layout.

\section{DISCUSSION}

Keystroke speed and accuracy are often used as performance measures for. evaluating computerbased writing/typing systems. Visual analysis of the KSPM figures indicates a consistent separation between performance on the default and QWERTY keyboard layout for Subjects 1 and 2 and a marginal separation for Subjects 3 and 4. Subjects 3 and 4 demonstrated an increasing trend in KSPM for both keyboards throughout the alternating treatment phases. All four subjects demonstrated a high degree of keystroke accuracy with both keyboard layouts, while their keystroke corrections varied in an irregular fashion.

Subjects 1 and 2 successfully used the LIAISON with the proportional-drive chin controller and default keyboard layout to complete their daily writing tasks prior to the study. They started at a visibly higher input rate on the default than Subjects 3 and 4 and maintained that higher level. During observation of the text entry trials it became evident that Subject 1 moved the cursor smoothly and accurately between characters, with minimal overshooting or rests between keystrokes when using the default layout. The potential ergonomic benefits of
Table 1.

Summary statistics for letter transition distances traveled on the default and QWERTY layouts for 50 most

frequently used words.

\begin{tabular}{|c|c|c|c|c|c|}
\hline \multirow{2}{*}{$\begin{array}{l}\text { Keyboard } \\
\text { Layour }\end{array}$} & \multicolumn{5}{|c|}{ Letter Transition Distance } \\
\hline & \multicolumn{2}{|l|}{ Mean } & \multicolumn{2}{|l|}{ Median } & $\mathrm{SD}$ \\
\hline Default & \multicolumn{2}{|l|}{2.58} & \multicolumn{2}{|l|}{2.24} & 1.09 \\
\hline \multirow[t]{2}{*}{ QWERTY } & \multicolumn{2}{|l|}{3.53} & \multicolumn{2}{|l|}{4.00} & 1.46 \\
\hline & \multicolumn{5}{|c|}{ Analysis of Variance } \\
\hline Source & df & $\begin{array}{l}\text { Sumi of } \\
\text { Squares }\end{array}$ & $\begin{array}{l}\text { Mean- } \\
\text { Square }\end{array}$ & T-Ratio & $\mathbf{P}$ \\
\hline $\begin{array}{l}\text { Keyboard } \\
\text { Layouts }\end{array}$ & 1 & 104.828 & 104.828 & 63.168 & .001 \\
\hline Error & 464 & 770.011 & 1.660 & & \\
\hline
\end{tabular}

the default appeared when the acceptance time was set at 16 and the user displayed skilled manipulation of the chin controller with the ability to move fluidly from letter to letter with few rests between characters and/or words.

The data for Subjects 3 and 4 demonstrates a gradual linear improvement in the ability to use default, suggesting some learning effect. This may have been due to several factors, including 1) mastering the use of the proportional chin controller, 2) adjusting to the scheduled increases in acceptance time, and, 3) learning the keyboard layouts. Subjects 3 and 4 had a total of 4 hours of practice on each keyboard over the course of the study. Both Subjects 3 and 4 had previous experience with the QWERTY keyboard layout, but no experience with the default. They obtained initial KSPM on the default that approached their experienced QWERTY level.

\section{Implications and conclusions}

The results across the four subjects suggest little difference in performance between the two keyboard layouts that cannot be explained by previous keyboard experience. Several factors must be considered when interpreting these results. For example, there appeared to be interference between the two keyboard layouts as they were alternated during the treatment sessions. All subjects reported more difficulty transferring from the QWERTY to the default within the initial treatment session. The possibility 
of a negative carryover effect across the trials is an area that requires further study and may have affected performance on both keyboards. Future investigations should include phases that do not change as rapidly as those included in the present study. A longer exposure to each of the keyboard arrangements may produce different results.

User fatigue may be a potentially confounding variable that must be addressed in conjunction with KSPM and KC in future studies. The amount of energy expended to achieve a maximum text entry rate is a factor in maintaining KSPM and $\mathrm{KC}$ and, therefore, may affect the overall long-term efficiency of the writing or typing system. Previous research on the QWERTY and alternative keyboards for touch typists and non-typists indicates that the distance traveled and the fatigue encountered are greater when using the QWERTY than for some of the ergonomically based replacements, such as the Dvorak (7). Traveling a greater distance between letters may result in increased fatigue if text entry periods are long. The text entry trials included in the present study were relatively short. A greater discrepancy between the two keyboards may have been demonstrated if the length of the test trials was significantly increased. This is an area that should be addressed in future investigations.

The $\mathrm{KC}$ is difficult to interpret due to substantial variability. A more comprehensive error analysis may provide insight regarding the importance of backspaces during text entry. This error analysis would involve developing a reliable way to record which keys were erased with the backspace and then replaced. The ability to correct errors using the backspace key confounds the measurement of rate and accuracy and should be incorporated into future studies of these performance characteristics. KC may be related to overshooting or undershooting the desired letter, irrespective of the keyboard. $\mathrm{KC}$ also may be related to lack of familiarity and expertise with the system, as KC was greater for the novice users.

Self-reports from Subjects 3 and 4 indicated that they worked "harder" with the QWERTY (moved the cursor more quickly/randomly) than with the default to obtain the input speeds recorded. Their statements were substantiated by the statistics on distance traveled (Table 1), which indicate that the user does travel a statistically greater distance using the QWERTY layout when compared with the default layout. Following the study, Subject 3 requested that the default keyboard layout be programmed into his present system, suggesting that he personally found this arrangement beneficial and easier to use. Additional research concerning user preference will be necessary to make appropriate recommendations concerning computer-based onscreen keyboard access systems.

Future research on the effectiveness of the different keyboard layouts should include more practice time on each system. The point of control for the chin controller or other interface device could also be an important variable in reaching maximum input speed. Additional research is clearly needed to examine the effectiveness of other interface devices. Another area of concern is the impact of an acceleration program on the efficiency of various keyboard arrangements. The optimal use of any keyboard layout for persons with high-level spinal cord injury will likely involve the use of an acceleration program.

The impact of alternative keyboard layouts on individual text entry performance requires more empirical attention. Only through future research will we be able to identify the potential of computerbased keyboard access systems to enhance the communication capabilities of persons with a disability.

\section{REFERENCES}

1. Demasco P, Oliviers D, Peterson W, Walsh C. The application of computer based workstations for vocational rehabilitation. Proceedings of ICAART; 1988 Jun 25-30; Montreal (QC). Washington (DC): RESNA, 1988: 39091 .

2. Horstmann HM, Levine SP, Kett RL. Commercial software as the basis for an augmentative communication system on a personal computer. Assist Technol 1990;2(1):19-26.

3. Horstmann HM, Levine SP. Modeling of user performance with computer access and augmentative communication systems for handicapped people. Augment Alternat Commun 1990;6:231-9.

4. Vanderheiden GC. Writing aids. In: Webster J, Cook A, Tompkins W, Vanderheiden G, editors. Electronic devices for rehabilitation. New York: John Wiley and Sons, 1985: 261-282.

5. Norman DA. The psychology of everyday things. New York: Basic Books, 1988. 
6. Kischenbaum A, Friedman Z, Melnik A. Performance of disabled persons on a chordic keyboard. Hum Factors 1986;28:187-94.

7. Cassingham RC. The Dvorak keyboard. Arcata (CA): Freelance Communications, 1986.

8. Bayer D, Nash R, Springsteen J. LIAISON by DU-IT: complete utilization of computers and software by high level SCI quadriplegic people. Proceedings of the RESNA 12th Annual Conference; 1989 Jun 25-30; New Orleans (LA). Washington (DC): RESNA Press, 1989: 345-6.

9. Alden DG, Daniels RW, Kanarick AF. Keyboard design and operation: a review of the major issues. Hum Factors 1972; 14:275-93.

10. Noyes J. The QWERTY keyboard: a review. Int J Man Mach Stud 1983;18:265-81.

11. DU-IT Control Systems Group, Inc. LIAISON instruction manual. Shreve (OH): The Group, 1988.

12. Workman D, Geggie C, Graham C. The microcomputer as an aid to written communication. Br J Occup Ther 1988;61:188-90.
13. Norman DA, Fisher D. Why alphabetic keyboards are not easy to use: keyboard layout doesn't much matter. Hum Factors 1982;24:509-19.

14. Angelo J, Deterdine $\mathrm{C}$, Weisman J, Comparing three head pointing systems using a single subject design. Assist Technol. In press.

15. Smith RO, Christiaansen R, Borden B, Lindberg D, Gunderson J, Vanderheiden G. Effectiveness of a writing system using a computerized long-range optical pointer and 10-branch abbreviation expansion. J Rehabil Res Dev 1989;26(1):51-62.

16. Kazdin AE. Single-case research designs: methods for clinical and applied settings. New York: Oxford Univ Press, 1982.

17. Ottenbacher KJ. Evaluating clinical change: strategies for occupational and physical therapists. Baltimore: Williams \& Wilkins, 1986.

18. Vanderheiden GC, Kelso DP. Comparative analysis of fixed-vocabulary communication acceleration techniques. Augment Alternat Commun 1987;3:196-206. 
